\title{
ABBREVIATIONS AND GLOSSARY
}

AI Artificial insemination

AIC African Inland Church

AIM African Inland Mission

ASAL Arid and Semi-Arid Lands program

CAIS Central Artificial Insemination Station

CBK Cooperative Bank of Kenya

CDC Commonwealth Development Corporation

DC District commissioner

DDC District Developmenc Committee

DDO District development officer

DVO District veterinary officer

EEC European Economic Community

GEMA Gikuyu, Embu, Meru Association

IADP Integrated Agricultural Development Project

ILO International Labor Organization

ILRAD International Laboratory for Research on Animal Disease

IMF International Monetary Fund

KADU Kenyan African Democratic Union

KANU Kenyan African National Union

KAU Kenyan African Union

KCC Kenya Cooperative Creameries

KDB Kenya Dairy Board

KETEPA Kenya Tea Packers, Ltd. 
KPU Kenya People's Union

KTDA Kenya Tea Development Authority

K.shs. Kenya shillings

MADO Maasai Agricultural Development Organization

MIDP Machakos Integrated Development Program

MIS Management information system

M.P. Member of Parliament

NCPB National Cereals and Produce Board

OPEC Organization of Petroleum Exporting Countries

PC Provincial commissioner

PCEA Presbyterian Church of East Africa

PS Permanent secretary

RDF Rural Development Fund

SRDP Special Rural Development Program

harambee self-help

majimbo regionalism

matajiri well-to-do 